\title{
Research Article \\ Exponential Stability for a Class of Stochastic Reaction-Diffusion Hopfield Neural Networks with Delays
}

\author{
Xiao Liang ${ }^{1}$ and Linshan Wang ${ }^{2}$ \\ ${ }^{1}$ College of Information Science and Engineering, Ocean University of China, Qingdao 266100, China \\ ${ }^{2}$ Department of Mathematics, Ocean University of China, Qingdao 266100, China
}

Correspondence should be addressed to Linshan Wang, wangls@ouc.edu.cn

Received 7 August 2011; Accepted 28 November 2011

Academic Editor: Jitao Sun

Copyright (C) 2012 X. Liang and L. Wang. This is an open access article distributed under the Creative Commons Attribution License, which permits unrestricted use, distribution, and reproduction in any medium, provided the original work is properly cited.

This paper studies the asymptotic behavior for a class of delayed reaction-diffusion Hopfield neural networks driven by finite-dimensional Wiener processes. Some new sufficient conditions are established to guarantee the mean square exponential stability of this system by using Poincarés inequality and stochastic analysis technique. The proof of the almost surely exponential stability for this system is carried out by using the Burkholder-Davis-Gundy inequality, the Chebyshev inequality and the Borel-Cantelli lemma. Finally, an example is given to illustrate the effectiveness of the proposed approach, and the simulation is also given by using the Matlab.

\section{Introduction}

Recently, the dynamics of Hopfield neural networks with reaction-diffusion terms have been deeply investigated because their various generations have been widely used in some practical engineering problems such as pattern recognition, associate memory, and combinatorial optimization (see [1-3]). However, under closer scrutiny, that a more realistic model would include some of the past states of the system, and theory of functional differential equations systems has been extensively developed [4, 5], meanwhile many authors have considered the asymptotic behavior of the neural networks with delays [69]. In fact random perturbation is unavoidable in any situation [3, 10]; if we include some environment noise in these systems, we can obtain a more perfect model of this situation 
$[3,11-16]$. So, this paper is devoted to the exponential stability of the following delayed reaction-diffusion Hopfield neural networks driven by finite-dimensional Wiener processes:

$$
\begin{aligned}
& d u_{i}(t, x)=\left(\sum_{j=1}^{l} \frac{\partial}{\partial x_{j}}\left(D_{i j}(x) \frac{\partial u_{i}}{\partial x_{j}}\right)-a_{i} u_{i}+\sum_{j=1}^{n} c_{i j} f_{j}\left(u_{j}(t-r, x)\right)\right) d t \\
& \quad+\sum_{j=1}^{m} g_{i j}\left(u_{i}(t-r, x)\right) d W_{j}, \\
&\left.\frac{\partial u_{i}}{\partial v}\right|_{\partial \mathcal{O}}=0, \quad t \geq 0, \\
& u_{i}(\theta, x)=\phi_{i}(\theta, x), \quad x \in \mathcal{O} \in \mathbb{R}^{l}, \theta \in[-r, 0], i=1,2, \ldots, n .
\end{aligned}
$$

There are $n$ neural network units in this system and $u_{i}(t, x)$ denote the potential of the cell $i$ at $t$ and $x . a_{i}$ are positive constants and denote the rate with which the $i$ th unit will reset its potential to the resting state in isolation when it is disconnected from the network and external inputs at $t$, and $c_{i j}$ are the output connection weights from the $j$ th neuron to the $i$ th neuron. $f_{j}$ are the active functions of the neural network. $r$ is the time delay of a neuron. $\mathcal{O}$ denotes an open bounded and connected subset of $\mathbb{R}^{l}$ with a sufficient regular boundary $\partial \mathcal{O}$, $v$ is the unit outward normal on $\partial \mathcal{O}, \partial u_{i} / \partial v=\left(\nabla u_{i}, v\right)_{\mathbb{R}^{l}}$, and $g_{i j}$ are noise intensities. Initial data $\phi_{i}$ are $\mathcal{F}_{0}$-measurable and bounded functions, almost surely.

We denote $(\Omega, \mathcal{F}, \mathbb{P})$ a complete probability space with filtration $\left\{\mathcal{F}_{t}\right\}_{t \geq 0}$ satisfying the usual conditions (see [10]). $W_{i}(t), i=1,2, \ldots, m$, are scale standard Brownian motions defined on $(\Omega, \mathcal{F}, \mathbb{P})$.

For convenience, we rewrite system (1.1) in the vector form:

$$
\begin{gathered}
d u=(\nabla \cdot(D(x) \circ \nabla u)-A u+C f(u(t-r))) d t+G(u(t-r)) d W, \\
\left.\frac{\partial u(t, x)}{\partial v}\right|_{\partial \mathcal{O}}=0, \quad t \geq 0, \\
u(0, x)=\phi(x),
\end{gathered}
$$

where $C=\left(c_{i j}\right)_{n \times n}, u=\left(u_{1}, u_{2}, \ldots, u_{n}\right)^{T}, \nabla u=\left(\nabla u_{1}, \ldots, \nabla u_{n}\right)^{T}, W=\left(W_{1}, W_{2}, \ldots, W_{m}\right)^{T}$, $f(u)=\left(f_{1}\left(u_{1}\right), f_{2}\left(u_{2}\right), \ldots, f_{n}\left(u_{n}\right)\right)^{T}, A=\operatorname{Diag}\left(a_{1}, a_{2}, \ldots, a_{n}\right), \phi=\left(\phi_{1}, \phi_{2}, \ldots, \phi_{n}\right)^{T}, G(u)=$ $\left(g_{i j}\left(u_{i}\right)\right)_{n \times m}, D=\left(D_{i j}\right)_{n \times l}$, and $D \circ \nabla u=\left(D_{i j} \partial u_{i} / \partial x_{j}\right)_{n \times l}$ is the Hadamard product of matrix $D$ and $\nabla u$; for the definition of divergence operator $\nabla \cdot u$, we refer to $[2,3]$.

\section{Preliminaries and Notations}

In this paper, we introduce the following Hilbert spaces $H \triangleq L^{2}(\mathcal{O}), V \triangleq H^{1}(\mathcal{O})$, according to [17-19], $V \subset H=H^{\prime} \subset V^{\prime}$, where $H^{\prime}, V^{\prime}$ denote the dual of the space $H, V$, respectively, the injection is continuous, and the embedding is compact. $\|\cdot\|,|\|\cdot \mid\|$ represent the norm in $H, V$, respectively.

$U \triangleq\left(L^{2}(\mathcal{O})\right)^{n}$ is the space of vector-valued Lebesgue measurable functions on $\mathcal{O}$, which is a Banach space under the norm $\|u\|_{U}=\left(\sum_{i=1}^{n}\left\|u_{i}(x)\right\|^{2}\right)^{1 / 2}$.

$C \triangleq C([-r, 0], U)$ is the Banach space of all continuous functions from $[-r, 0]$ to $U$, when equipped with the sup-norm $\|\phi\|_{C}=\sup _{-r \leq s \leq 0}\|\phi\|_{U}$. 
With any continuous $\mathcal{F}_{t}$-adapted $U$-valued stochastic process $u(t): \Omega \rightarrow U, t \geq-r$, we associate a continuous $\mathcal{F}_{t}$-adapted $C$-valued stochastic process $u_{t}: \Omega \rightarrow C, t>0$, by setting $u_{t}(s, x)(\omega)=u(t+s, x)(\omega), s \in[-r, 0], x \in \mathcal{O}$.

$C_{q_{0}}^{b}$ denote the space of all bounded continuous processes $\phi:[-r, 0] \times \Omega \rightarrow U$ such that $\phi(\theta, \cdot)$ is $\mathcal{F}_{0}$-measurable for each $\theta \in[-r, 0]$ and $E\|\phi\|_{C}<\infty$.

$\mathcal{L}(K)$ is the set of all linear bounded operators from $K$ into $K$; when equipped with the operator norm, it becomes a Banach space.

In this paper, we assume the following.

H1 $f_{i}$ and $G_{i j}$ are Lipschitz continuous with positive Lipschitz constants $k_{1}, k_{2}$ such that $\left|f_{i}(u)-f_{i}(v)\right| \leq k_{1}|u-v|$ and $\left|G_{i j}(u)-G_{i j}(v)\right| \leq k_{2}|u-v|, \forall u, v \in \mathbb{R}$, and $f_{i}(0)=0$, $g_{i j}(0)=0$.

H2 There exists $\alpha>0$ such that $D_{i j}(x) \geq \alpha / l$.

H3 Let $\eta=2 \alpha \beta^{2}+2 k_{3}-n k_{1}^{2} \sigma^{2} e^{r}-m k_{2}^{2} e^{r}-2>0, k_{3}=\min \left\{a_{i}\right\}, \sigma=\max \left\{\left|c_{i j}\right|\right\}$.

Remark 2.1. We can infer from $\mathrm{H} 1$ that system (1.1) has an equilibrium $u(t, x, \omega)=0$.

Let us define the linear operator as follows:

$$
\begin{array}{r}
\mathfrak{A}: \Pi(\mathfrak{A}) \in U \longrightarrow U, \\
\mathfrak{A} u=\nabla \cdot(D(x) \circ \nabla u),
\end{array}
$$

and $\Pi(\mathfrak{A})=\left\{u \in H^{2}(\mathcal{O})^{n}, \partial u /\left.\partial v\right|_{\partial \mathcal{O}}=0\right\}$

Lemma 2.2 (Poincaré's inequality). Let $\mathcal{O}$ be a bounded domain in $R^{l}$ and $\phi$ belong to a collection of twice differentiable functions defined on $\mathcal{O}$ into $R$; then

$$
\|\phi\| \leq \beta^{-1}|\|\phi\||
$$

where the constant $\beta$ depends on the size of $\mathcal{O}$.

Lemma 2.3. Let us consider the equation

$$
\begin{gathered}
\frac{d u}{d t}=\mathfrak{A} u, \quad t \geq 0, \\
u(0)=\phi .
\end{gathered}
$$

For every $\phi \in U$, let $u(t)=S(t) \phi$ denote the solution of $(2.3)$; then $S(t)$ is a contraction map in $U$.

Proof. Now we take the inner product of (2.3) with $u(t)$ in $U$; by employing the Gaussian theorem and condition $\mathrm{H} 2$, we get that $(\mathfrak{A} u, u) \leq-\alpha\|\| u \|_{H^{1}(\mathcal{O})^{n}}^{2}(\cdot, \cdot)$ is the inner product in $U$, $\||u|\|_{H^{1}(\mathcal{O})^{n}}^{2}$ denote the norm of $H^{1}(\mathcal{O})^{n}$ (see [3]), which means

$$
\frac{1}{2} \frac{d}{d t}\|u(t)\|_{U}^{2}+\alpha\|\| u(t) \|_{H^{1}(\mathcal{O})^{n}}^{2} \leq 0
$$


Thanks to the Poincaré inequality, one obtains

$$
\frac{d}{d t}\|u(t)\|_{U}^{2}+2 \alpha \beta^{2}\|u(t)\|_{U}^{2} \leq 0
$$

Multiplying $e^{2 \alpha \beta^{2} t}$ in both sides of the inequality, we have

$$
\frac{d}{d t}\left(e^{2 \alpha \beta^{2} t}\|u(t)\|_{U}^{2}\right) \leq 0
$$

Integrating the above inequality from 0 to $t$, we obtain

$$
\|u(t)\|_{U}^{2} \leq e^{-2 \alpha \beta^{2} t}\|\phi\|_{U}^{2}
$$

By the definition of $\|T(t)\|_{\mathcal{L}(U)}$, we have $\|T(t)\|_{\mathcal{L}(U)} \leq 1$.

Definition 2.4 (see [20-22]). A stochastic process $u(t):[-r,+\infty) \times \Omega \rightarrow U$ is called a global mild solution of (1.1) if

(i) $u(t)$ is adapted to $\mathcal{F}_{t}$

(ii) $u(t)$ is measurable with $\int_{0}^{\infty}\|u(t)\|_{U}^{2} d t<\infty$ almost surely and

$$
\begin{aligned}
& u(t)=S(t) \phi-\int_{0}^{t} S(t-s) \mathrm{Ads}+\int_{0}^{t} S(t-s) f(u(s-r)) d s+\int_{0}^{t} S(t-s) G(u(s-r)) d W, \\
& u(t)=\phi \in C_{\bar{\mp}_{0}^{\prime}}^{b} \quad t \in[-r, 0]
\end{aligned}
$$

for all $t \in[-r,+\infty)$ with probability one.

Definition 2.5. Equation (1.1) is said to be almost surely exponentially stable if, for any solution $u(t, x, \omega)$ with initial data $\phi \in C_{\mathcal{F}_{0}}^{b}$, there exists a positive constant $\lambda$ such that

$$
\limsup _{t \rightarrow \infty} \ln \left\|u_{t}\right\|_{C} \leq-\lambda, \quad u_{t} \in C, \text { almost surely. }
$$

Definition 2.6. System (1.1) is said to be exponentially stable in the mean square sense if there exist positive constants $\kappa$ and $\alpha$ such that, for any solution $u(t, x, \omega)$ with the initial condition $\phi \in C_{q_{0}}^{b}$, one has

$$
E\|u(t)\|_{C}^{2} \leq \kappa e^{-\alpha\left(t-t_{0}\right)}, \quad t \geq t_{0}, u_{t} \in C
$$

\section{Main Result}

Theorem 3.1. Suppose conditions H1-H3 hold; then (1.1) is exponentially stable in the mean square sense. 
Proof. Let $u$ be the mild solution of (1.1); thanks to the Itô formula, we observe that

$$
\begin{aligned}
d\left(e^{\lambda t} u_{i}^{2}\right)= & \lambda e^{\lambda t} u_{i}^{2} d t+e^{\lambda t}\left(2 u_{i}\left(\sum_{j=1}^{l} \frac{\partial}{\partial x_{i}}\left(D_{i j} \frac{\partial u_{i}}{\partial x_{j}}\right)-a_{i} u_{i}+\sum_{j=1}^{n} c_{i j} f_{j}\left(u_{j}(t-r)\right)\right)\right) d t \\
& +e^{\lambda t}\left(G_{i} G_{i}^{T}\right) d t+2 e^{\lambda t} u_{i} G_{i} d W, \quad G_{i}=\left(G_{i 1}, G_{i 2}, \ldots, G_{i m}\right)
\end{aligned}
$$

where $\lambda$ is a positive constant that will be defined below. Then, by integration between 0 and $t$, we find that

$$
\begin{aligned}
e^{\lambda t} u_{i}^{2}(t)= & \phi_{i}(0)^{2}+\int_{0}^{t} \lambda e^{\lambda s} u_{i}^{2} d s+2 \int_{0}^{t} e^{\lambda s}\left(u_{i} \sum_{j=1}^{l} \frac{\partial}{\partial x_{j}}\left(D_{i j} \frac{\partial u_{i}}{\partial x_{j}}\right)\right) d s-2 \int_{0}^{t} e^{\lambda s} a_{i} u_{i}^{2} d s \\
& +2 \int_{0}^{t} e^{\lambda s} u_{i} \sum_{j=1}^{n} c_{i j} f_{j}\left(u_{j}(s-r)\right) d s+\int_{0}^{t} e^{\lambda s} G_{i} G_{i}^{T} d s+2 \int_{0}^{t} e^{\lambda s} u_{i} G_{i} d W
\end{aligned}
$$

Integrating the above equation over $\mathcal{O}$, by virtue of Fubini's theorem, we prove that

$$
\begin{aligned}
e^{\lambda t}\left\|u_{i}^{2}\right\|^{2}= & \left\|\phi_{i}(0)\right\|^{2}+\lambda \int_{0}^{t} e^{\lambda s} \int_{\mathcal{O}} u_{i}^{2} d x d s+\int_{0}^{t} e^{\lambda s} \int_{\mathcal{O}} 2 u_{i} \sum_{j=1}^{l} \frac{\partial}{\partial x_{j}}\left(D_{i j} \frac{\partial u_{i}}{\partial x_{j}}\right) d x d s \\
& -2 \int_{0}^{t} e^{\lambda s} \int_{\mathcal{O}} a_{i} u_{i}^{2} d x d s+2 \int_{0}^{t} e^{\lambda s} \int_{\mathcal{O}} u_{i} \sum_{j=1}^{n} c_{i j} f_{j}\left(u_{j}(s-r)\right) d x d s \\
& +\int_{0}^{t} e^{\lambda s} \int_{\mathcal{O}} G_{i} G_{i}^{T} d x d s+2 \int_{0}^{t} e^{\lambda s} \int_{\mathcal{O}} u_{i} G_{i} d x d W
\end{aligned}
$$

Taking the expectation on both sides of the last equation, by means of $[3,10,16]$

$$
2 E \int_{0}^{t} \int_{\mathcal{O}} e^{\lambda s} u_{i} G_{i} d x d W=0
$$

Then, by Fubini's theorem, we have

$$
\begin{aligned}
e^{\lambda t} E\left\|u_{i}^{2}\right\|^{2}= & E\left\|\phi_{i}(0)\right\|^{2}+\lambda \int_{0}^{t} e^{\lambda s} \int_{\mathcal{O}} E u_{i}^{2} d x d s+2 E \int_{0}^{t} e^{\lambda s} \int_{\mathcal{O}} u_{i} \sum_{j=1}^{l} \frac{\partial}{\partial x_{j}}\left(D_{i j} \frac{\partial u_{i}}{\partial x_{j}}\right) d x d s \\
& -2 \int_{0}^{t} e^{\lambda s} \int_{\mathcal{O}} a_{i} E u_{i}^{2} d x d s+2 E \int_{0}^{t} e^{\lambda s} \int_{\mathcal{O}} u_{i} \sum_{j=1}^{n} c_{i j} f_{j}\left(u_{j}(s-r)\right) d x d s \\
& +E \int_{0}^{t} e^{\lambda s} \int_{\mathcal{O}} G_{i} G_{i}^{T} d x d s \\
\triangleq & I_{1}+I_{2}+I_{3}+I_{4}+I_{5}+I_{6} .
\end{aligned}
$$


We observe that

$$
\begin{gathered}
I_{1} \triangleq E\left\|\phi_{i}(0)\right\|^{2} \leq \sup _{\theta \in[-r, 0]} E\left\|\phi_{i}(\theta)\right\|^{2}, \\
I_{2} \triangleq \lambda \int_{0}^{t} \int_{\mathcal{O}} e^{\lambda s} E u_{i}^{2} d x d s=\lambda \int_{0}^{t} e^{\lambda s} E\left\|u_{i}\right\|^{2} d s .
\end{gathered}
$$

From the Neumann boundary condition, by means of Green's formula and H2 (see $[3,6,7])$, we know

$$
\begin{aligned}
I_{3} & \triangleq 2 E \int_{0}^{t} \int_{\mathcal{O}} e^{\lambda s}\left(u_{i} \sum_{j=1}^{l} \frac{\partial}{\partial x_{j}}\left(D_{i j} \frac{\partial u_{i}}{\partial x_{j}}\right)\right) d x d s \\
& =-2 E \int_{0}^{t} \int_{\mathcal{O}} e^{\lambda s} \sum_{j=1}^{l} D_{i j}\left(\frac{\partial u_{i}}{\partial x_{j}}\right)^{2} d x d s \\
& \leq-2 \alpha \int_{0}^{t} e^{\lambda s} E \mid\left\|u_{i}\right\|^{2} d s \leq-2 \alpha \beta^{2} \int_{0}^{t} e^{\lambda s} E\left\|u_{i}\right\|^{2} d s .
\end{aligned}
$$

Then, by using the positiveness of $a_{i}$, one gets the relation

$$
I_{4} \triangleq-2 \int_{0}^{t} \int_{\mathcal{O}} e^{\lambda s} a_{i} E u_{i}^{2} d x d s \leq-2 k_{3} \int_{0}^{t} e^{\lambda s} E\left\|u_{i}\right\|^{2} d s,
$$

where $k_{3}=\min \left\{a_{1}, a_{2}, \ldots, a_{n}\right\}>0$. By using the Young inequality as well as condition H1, we have that

$$
\begin{aligned}
I_{5} & \triangleq 2 E \int_{0}^{t} \int_{\mathcal{O}} e^{\lambda s} u_{i} \sum_{j=1}^{n} c_{i j} f_{j} d x d s \\
& \leq \int_{0}^{t} \int_{\mathcal{O}} e^{\lambda s}\left(E\left|u_{i}\right|^{2}+E\left|\sum_{j=1}^{n} c_{i j} f_{j}\right|^{2}\right) d x d s \\
& \leq \int_{0}^{t} \int_{\mathcal{O}} e^{\lambda s}\left(E\left|u_{i}\right|^{2}+\sigma^{2} \sum_{j=1}^{n} E\left|f_{j}\left(u_{j}(s-r)\right)\right|^{2}\right) d x d s \\
& \leq \int_{0}^{t} \int_{\mathcal{O}} e^{\lambda s}\left(E\left|u_{i}\right|^{2}+\sigma^{2} k_{1}^{2} \sum_{j=1}^{n} E\left|u_{j}(s-r)\right|^{2}\right) d x d s \\
& \leq \int_{0}^{t} e^{\lambda s}\left(E\left\|u_{i}\right\|^{2}+\sigma^{2} k_{1}^{2} E\|u(s-r)\|_{u}^{2}\right) d s,
\end{aligned}
$$

where $\sigma=\max \left|c_{i j}\right|$, and

$$
I_{6} \triangleq \int_{0}^{t} \int_{\mathcal{O}} e^{\lambda s} E G_{i} G_{i}^{T} d x d s \leq m k_{2}^{2} \int_{0}^{t} e^{\lambda s} E\left\|u_{i}(s-r)\right\|^{2} d s .
$$


We infer from (3.6)-(3.11) that

$$
\begin{aligned}
e^{\lambda t} E\left\|u_{i}(t)\right\|^{2} \leq & \sup _{\theta \in[-r, 0]} E\left\|\phi_{i}(\theta)\right\|^{2}-\left(2 \alpha \beta^{2}+2 k_{3}-1-\lambda\right) \int_{0}^{t} e^{\lambda s} E\left\|u_{i}\right\|^{2} d s \\
& +\sigma^{2} k_{1}^{2} \int_{0}^{t} e^{\lambda s}\|u(t-r)\|_{U}^{2} d s+m k_{2}^{2} \int_{0}^{t} e^{\lambda s} E\left\|u_{i}(s-r)\right\|^{2} d s .
\end{aligned}
$$

Adding (3.12) from $i=1$ to $i=n$, we obtain

$$
\begin{aligned}
e^{\lambda t} E\|u\|_{U}^{2} \leq & E\|\phi\|_{C}^{2}-\left(2 \alpha \beta^{2}+2 k_{3}-1-\lambda\right) \int_{0}^{t} e^{\lambda s} E\|u\|_{U}^{2} d s \\
& +\left(n k_{1}^{2} \sigma^{2}+m k_{2}^{2}\right) \int_{0}^{t} e^{\lambda s} E\|u(s-r)\|_{U}^{2} d s
\end{aligned}
$$

due to

$$
\begin{aligned}
\int_{0}^{t} e^{\lambda s} E\|u(s-r)\|_{U}^{2} d s & \leq e^{\lambda r} \int_{-r}^{t} e^{\lambda s} E\|u(s)\|_{U}^{2} d s \\
& \leq e^{2 \lambda r} \int_{-r}^{0} E\|\phi(s)\|_{U}^{2} d s+e^{\lambda r} \int_{0}^{t} e^{\lambda s} E\|u(s)\|_{U}^{2} d s \\
& \leq r e^{2 \lambda r} E\|\phi\|_{C}^{2}+e^{\lambda r} \int_{0}^{t} e^{\lambda s} E\|u(s)\|_{U}^{2} d s
\end{aligned}
$$

we induce from the previous equations that

$$
e^{\lambda t} E\|u\|_{U}^{2} \leq-c_{1} \int_{0}^{t} e^{\lambda s} E\|u\|_{U}^{2} d s+c_{2}
$$

where $c_{1}=2 \alpha \beta^{2}+2 k_{3}-1-n k_{1}^{2} \sigma^{2} e^{\lambda r}-m k_{2}^{2} e^{\lambda r}-\lambda$ and $c_{2}=\left(1+m k_{2}^{2} r e^{2 \lambda r}+n k_{1}^{2} \sigma^{2} r e^{2 \lambda r}\right) E\|\phi\|_{C}^{2}$; so we choose $\lambda=1$ such that $c_{1}=\eta>0$. By using the classical Gronwall inequality we see that

$$
e^{\lambda t} E\|u\|_{U}^{2} \leq c_{2} e^{-\eta t}
$$

in other words, we get

$$
E\|u\|_{U}^{2} \leq c_{2} e^{-(\eta+1) t} .
$$

So, for $t+\theta \geq t / 2 \geq 0$, we also have

$$
\begin{aligned}
E\|u(t+\theta)\|_{U}^{2} & \leq c_{2} e^{-(\eta+1)(t+\theta)}, \\
& \leq c_{2} e^{-\kappa t}, \quad \theta \in[-r, 0], \kappa=\frac{(\eta+1)}{2},
\end{aligned}
$$


and we can conclude that

$$
E\left\|u_{t}\right\|_{C}^{2} \leq c_{2} e^{-\kappa t}
$$

Theorem 3.2. If the system (1.1) satisfies hypotheses H1-H3, then it is almost surely exponentially stable.

Proof. Let $u(t)$ be the mild solution of (1.1). By Definition 2.4 as well as the inequality $\left(\sum_{i=1}^{n} a_{i}\right)^{2} \leq n \sum_{i=1}^{n} a_{i}^{2}, a_{i} \in \mathbb{R}$, we have

$$
\begin{aligned}
E \sup _{N \leq t \leq N+1}\|u(t)\|_{U}^{2} \leq & 4 \sup _{N \leq t \leq N+1}\|S(t-N+1) u(N)\|_{U}^{2} \\
& +4 \sup _{N \leq t \leq N+1}\left\|\int_{N-1}^{t}-A S(t-s) u d s\right\|_{U}^{2} \\
& +4 \sup _{N \leq t \leq N+1}\left\|\int_{N-1}^{t} S(t-s) C f(u(s-r)) d s\right\|_{U}^{2} \\
& +4 \sup _{N \leq t \leq N+1}\left\|\int_{N-1}^{t} S(t-s) G(u(s-r)) d W\right\|_{U}^{2} \\
\triangleq & I_{1}+I_{2}+I_{3}+I_{4} .
\end{aligned}
$$

Using the contraction of the map $S(t)$ and the result of Theorem 3.1, we find

$$
\begin{aligned}
I_{1} & \triangleq 4 \sup _{N \leq t \leq N+1} E\|(S(t-N+1) u(N-1))\|_{U}^{2} \\
& \leq 4 \sup _{N \leq t \leq N+1} E\left\|u_{N-1}\right\|_{C}^{2} \leq 4 c_{2} e^{-\kappa(N-1)} .
\end{aligned}
$$

By the Hölder inequality, we obtain

$$
\begin{aligned}
I_{2} & \triangleq 4 \sup _{N \leq t \leq N+1} E\left\|\int_{N-1}^{t}-A S(t-s) u d s\right\|_{U}^{2} \\
& \leq 4 \sup _{N \leq t \leq N+1}(t-N+1) \int_{N-1}^{t} E\|-A S(t-s) u\|_{U}^{2} d s \\
& \leq 8 \sup _{N \leq t \leq N+1} \int_{N-1}^{t} E\|A u\|_{U}^{2} d s \\
& \leq 8 k_{4}^{2} \int_{N-1}^{N+1} E\|u\|_{U}^{2} d s \leq 8 k_{4}^{2} \int_{N-1}^{N+1} E\left\|u_{s}\right\|_{C}^{2} d s \\
& \leq 8 k_{4}^{2} c_{2} \int_{N-1}^{N+1} e^{-\kappa s} d s \leq 8 k_{4}^{2} \rho_{1} e^{-\kappa(N-1)}
\end{aligned}
$$

where $\rho_{1}=c_{2} / \kappa, k_{4}=\max \left\{a_{1}, a_{2}, \ldots, a_{n}\right\}$. 
By virtue of Theorem 3.1, Hölder inequality, and H1, we have

$$
\begin{aligned}
I_{3} & \triangleq 4 \sup _{N \leq t \leq N+1}\left\|E \int_{N-1}^{t} S(t-s) C f(u(s-r)) d s\right\|_{U}^{2} \\
& \leq 4 \sup _{N \leq t \leq N+1}(t-N+1) E \int_{N-1}^{t}\|C f(u(s-r))\|_{U}^{2} d s \\
& \leq 8 \sigma^{2} \sup _{N \leq t \leq N+1} E \int_{N-1}^{t}\|f(u(s-r))\|_{U}^{2} d s \\
& \leq 8 k_{1}^{2} \sigma^{2} \int_{N-1}^{N+1} E\|u(s-r)\|_{U}^{2} d s \leq 8 k_{1}^{2} \sigma^{2} \int_{N-1}^{N+1} E\left\|u_{s}\right\|_{C}^{2} d s \\
& \leq 8 k_{1}^{2} c_{2} \int_{N-1}^{N+1} e^{-\kappa s} d s \leq 8 k_{1}^{2} \rho_{1} e^{-\kappa(N-1)} .
\end{aligned}
$$

Then, by the Burkholder-Davis-Gundy inequality (see $[18,22])$, there exists $c_{3}$ such that

$$
\begin{aligned}
I_{4} & \triangleq 4 \sup _{N \leq t \leq N+1} E\left\|\int_{N-1}^{t} S(t-s) G(u(s-r)) d W\right\|_{U}^{2} \\
& \leq 4 c_{3} \sup _{N \leq t \leq N+1} E \int_{N-1}^{t}\|S(t-s) G(u(s-r)) I\|_{U}^{2} d s \\
& \leq 4 c_{3} k_{2}^{2} \sup _{N \leq t \leq N+1} \int_{N-1}^{t} E\|u(s-r)\|_{U}^{2} d s \leq 4 c_{3} k_{2}^{2} \int_{N-1}^{N+1} E\left\|u_{s}\right\|_{C}^{2} d s \\
& \leq 4 c_{3} k_{2}^{2} c_{2} \int_{N-1}^{N+1} e^{-\kappa s} d s \leq 4 c_{3} k_{2}^{2} \rho_{1} e^{-\kappa(N-1)}
\end{aligned}
$$

where $I=(1,1, \ldots, 1)^{T}$ is an $m$-dimensional vector.

We can deduce from (3.21)-(3.24) that

$$
E \sup _{N \leq t \leq N+1}\|u(t)\|_{U}^{2} \leq \rho_{2} e^{-\kappa(N-1)}
$$

where $\rho_{2}=4 c_{2}+\left(8 k_{4}^{2}+8 k_{1}^{2}+4 c_{3} k_{2}^{2}\right) \rho_{1}$.

Thus, for any positive constants $\varepsilon_{N}$, thanks to the Chebyshev inequality we have that

$$
\begin{aligned}
P\left(\sup _{N \leq t \leq N+1}\|u(t)\|_{U}>\varepsilon_{N}\right) & \leq \frac{1}{\varepsilon_{N}^{2}} \sup _{N \leq t \leq N+1} E\|u(t)\|_{U}^{2} \\
& \leq \frac{1}{\varepsilon_{N}^{2}} \rho_{2} e^{-\kappa(N-1)}
\end{aligned}
$$




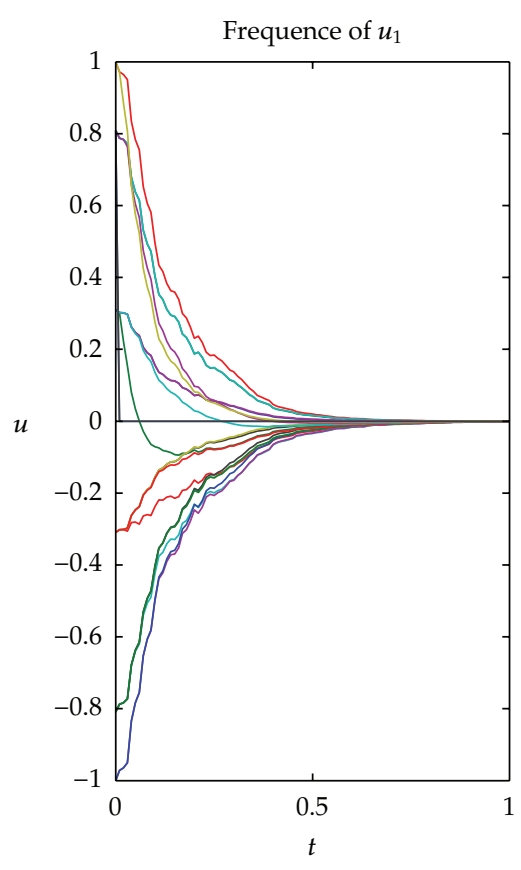

(a)

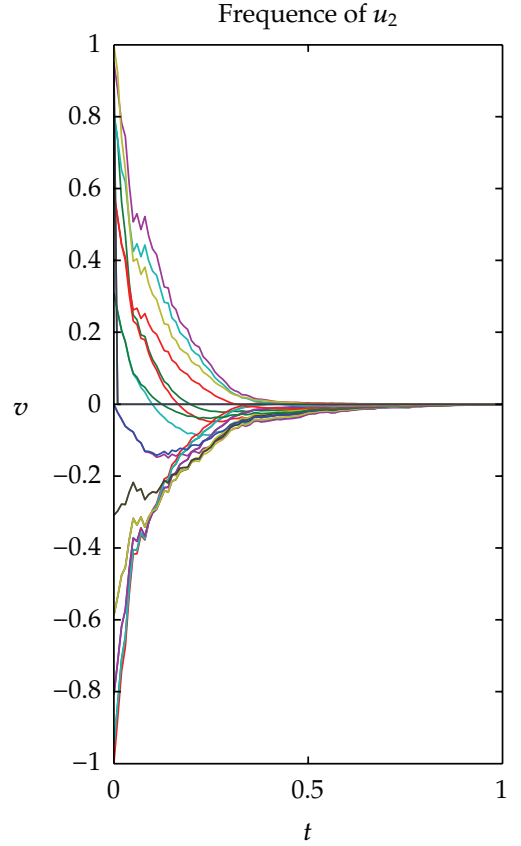

(b)

Figure 1

Due to the Borel-Cantelli lemma, we see that

$$
\limsup _{t \rightarrow \infty} \frac{\ln \|u(t)\|_{U}^{2}}{t} \leq-\kappa, \quad \text { almost surely. }
$$

This completes the proof of the theorem.

\section{Simulation}

Consider two-dimensional stochastic reaction-diffusion recurrent neural networks with delay as follows:

$$
\begin{aligned}
d u_{1}(t, x)= & \left(10 \Delta u_{1}-7 u_{1}+1.3 \tanh \left(u_{1}(t-1, x)\right)\right) d t+u_{1}(t-1, x) d W \\
d u_{2}(t, x)= & \left(10 \Delta u_{2}-7 u_{2}+\tanh \left(u_{1}(t-1, x)\right)-\tanh \left(u_{2}(t-1, x)\right)\right) d t \\
& +u_{2}(t-1, x) d W \\
\frac{\partial u_{i}(t, 0)}{\partial x}= & \frac{\partial u_{i}(t, 20)}{\partial x}=0, \quad t \geq 0, \\
u_{1}(\theta, x)= & \cos (0.2 \pi x), \quad u_{2}(\theta, x)=\cos (0.1 \pi x), x \in[0,20], \theta \in[-1,0] .
\end{aligned}
$$

$\Delta$ is the Laplace operator. We have $\beta \geq 1 / 20, \alpha \geq 10, k_{1}=1, k_{2}=1, k_{3}=7, \sigma=1.3, n=2$, and $\eta>0$; by Theorems 3.1 and 3.2, this system is mean square exponentially stable as well as almost surely exponentially stable. The results can be shown in Figures 1, 2 and 3. 


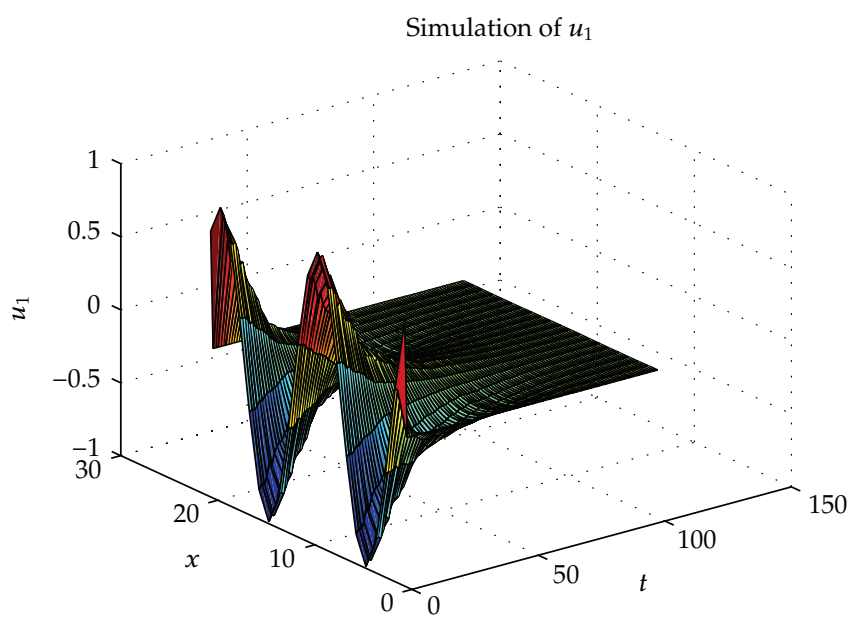

Figure 2

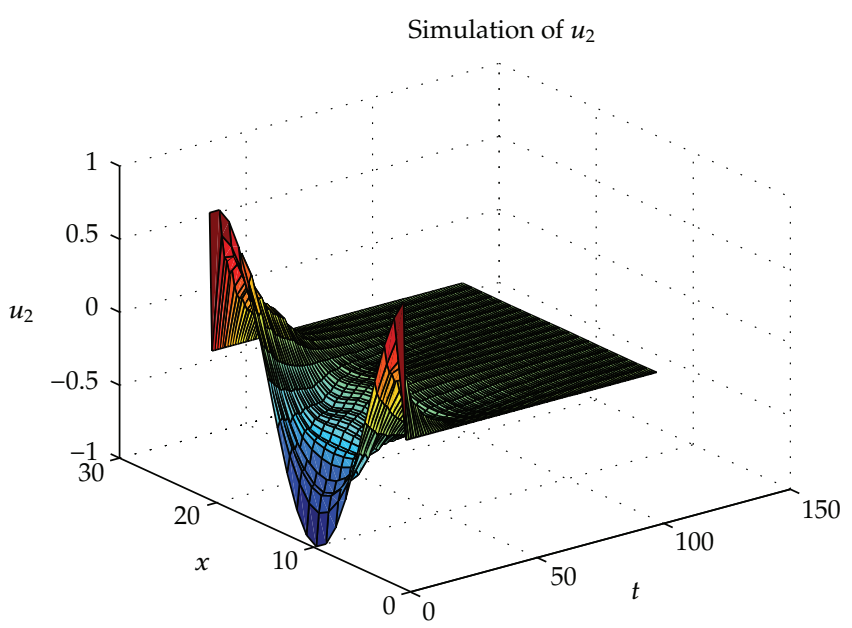

Figure 3

We use the forward Euler method to simulate this example [23-25]. We choose the time step $\Delta t=0.01$ and space step $\Delta x=1$, and $\delta=\Delta t / \Delta x^{2}=0.01$.

\section{Acknowledgment}

The authors wish to thank the referees for their suggestions and comments. We are also indebted to the editors for their help. This work was supported by the National Natural Science Foundation of China (no. 11171374), Natural Science Foundation of Shandong Province (no. ZR2011AZ001). 


\section{References}

[1] X. X. Liao and Y. L. Gao, "Stability of Hopfield neural networks with reactiondiffusion terms," Acta Electronica Sinica, vol. 28, pp. 78-82, 2000.

[2] L. S. Wang and D. Xu, "Global exponential stability of Hopfield reaction-diffusion neural networks with time-varying delays," Science in China F, vol. 46, no. 6, pp. 466-474, 2003.

[3] L. S. Wang and Y. F. Wang, "Stochastic exponential stability of the delayed reaction diffusion interval neural networks with Markovian jumpling parameters," Physics Letters A, vol. 356, pp. 346-352, 2008.

[4] J. K. Hale and V. S. M. Lunel, Introduction to Functional-Differential Equations, vol. 99 of Applied Mathematical Sciences, Springer, Berlin, Germany, 1993.

[5] S.-E. A. Mohammed, Stochastic Functional Differential Equations, vol. 99 of Research Notes in Mathematics, Pitman, London, UK, 1984.

[6] L. S. Wang and Y. Y. Gao, "Global exponential robust stability of reactiondiffusion interval neural networks with time varying delays," Physics Letters A, vol. 305, pp. 343-348, 2006.

[7] L. S. Wang, R. Zhang, and Y. Wang, "Global exponential stability of reaction-diffusion cellular neural networks with S-type distributed time delays," Nonlinear Analysis: Real World Applications, vol. 10, no. 2, pp. 1101-1113, 2009.

[8] H. Y. Zhao and G. L. Wang, "Existence of periodic oscillatory solution of reactiondiffusion neural networks with delays," Physics Letters A, vol. 343, pp. 372-382, 2005.

[9] J. G. Lu and L. J. Lu, "Global exponential stability and periodicity of reaction-diffusion recurrent neural networks with distributed delays and Dirichlet boundary conditions," Chaos, Solitons and Fractals, vol. 39, no. 4, pp. 1538-1549, 2009.

[10] X. Mao, Stochastic Differential Equations and Applications, Horwood, 1997.

[11] J. Sun and L. Wan, "Convergence dynamics of stochastic reaction-diffusion recurrent neural networks with delays," International Journal of Bifurcation and Chaos in Applied Sciences and Engineering, vol. 15, no. 7, pp. 2131-2144, 2005.

[12] M. Itoh and L. O. Chua, "Complexity of reaction-diffusion CNN," International Journal of Bifurcation and Chaos in Applied Sciences and Engineering, vol. 16, no. 9, pp. 2499-2527, 2006.

[13] X. Lou and B. Cui, "New criteria on global exponential stability of BAM neural networks with distributed delays and reaction-diffusion terms," International Journal of Neural Systems, vol. 17, pp. 43-52, 2007.

[14] Q. Song and Z. Wang, "Dynamical behaviors of fuzzy reaction-diffusion periodic cellular neural networks with variable coefficients and delays," Applied Mathematical Modelling, vol. 33, no. 9, pp. 3533-3545, 2009.

[15] Q. Song, J. Cao, and Z. Zhao, "Periodic solutions and its exponential stability of reaction-diffusion reccurent neural networks with distributed time delays," Nonlinear Analysis B, vol. 8, pp. 345-361, 2007.

[16] K. Liu, "Lyapunov functionals and asymptotic stability of stochastic delay evolution equations," Stochastics and Stochastics Reports, vol. 63, no. 1-2, pp. 1-26, 1998.

[17] R. Temam, Infinite-Dimensional Dynamical Systems in Mechanics and Physics, vol. 68 of Applied Mathematical Sciences, Springer, New York, NY, USA, 1988.

[18] G. Da Prato and J. Zabczyk, Stochastic Equations in Infinite Dimensions, vol. 44 of Encyclopedia of Mathematics and its Applications, Cambridge University Press, Cambridge, UK, 1992.

[19] I. Chueshov, Introduction to the Theory of Infinite-Dimensional Dissipative Systems, Acta Kharkov, 2002.

[20] R. Jahanipur, "Stochastic functional evolution equations with monotone nonlinearity: existence and stability of the mild solutions," Journal of Differential Equations, vol. 248, no. 5, pp. 1230-1255, 2010.

[21] T. Taniguchi, "Almost sure exponential stability for stochastic partial functional-differential equations," Stochastic Analysis and Applications, vol. 16, no. 5, pp. 965-975, 1998.

[22] T. Caraballo, K. Liu, and A. Truman, "Stochastic functional partial differential equations: existence, uniqueness and asymptotic decay property," Proceedings the Royal Society of London A, vol. 456, no. 1999, pp. 1775-1802, 2000.

[23] M. Kamrani and S. M. Hosseini, "The role of coefficients of a general SPDE on the stability and convergence of a finite difference method," Journal of Computational and Applied Mathematics, vol. 234, no. 5, pp. 1426-1434, 2010.

[24] D. J. Higham, "Mean-square and asymptotic stability of the stochastic theta method," SIAM Journal on Numerical Analysis, vol. 38, no. 3, pp. 753-769, 2000.

[25] P. E. Kloeden and E. Platen, Numerical Solution of Stochastic Differential Equations, vol. 23, Springer, Berlin, Germany, 3rd edition, 1999. 


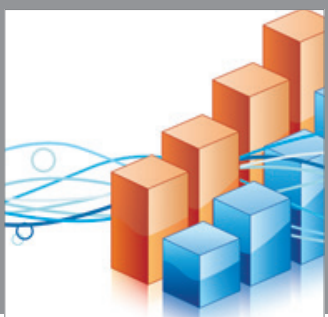

Advances in

Operations Research

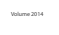

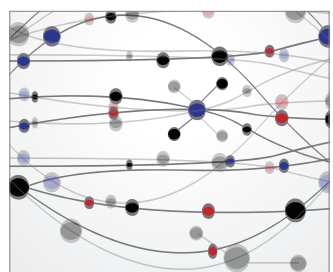

\section{The Scientific} World Journal
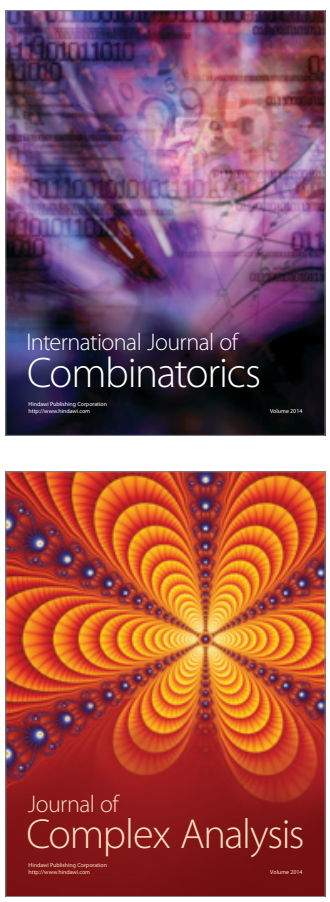

International Journal of

Mathematics and

Mathematical

Sciences
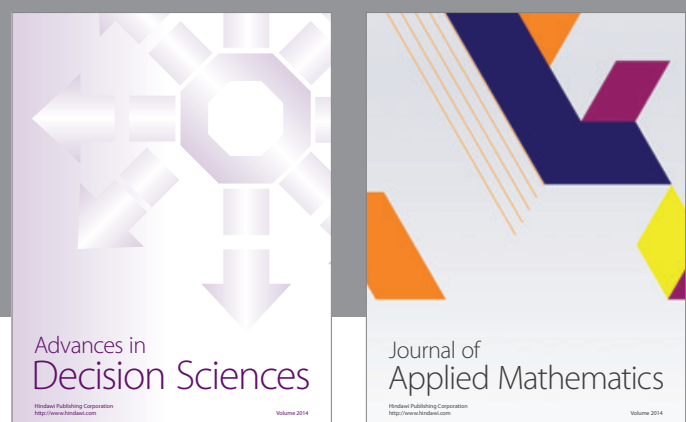

Journal of

Applied Mathematics
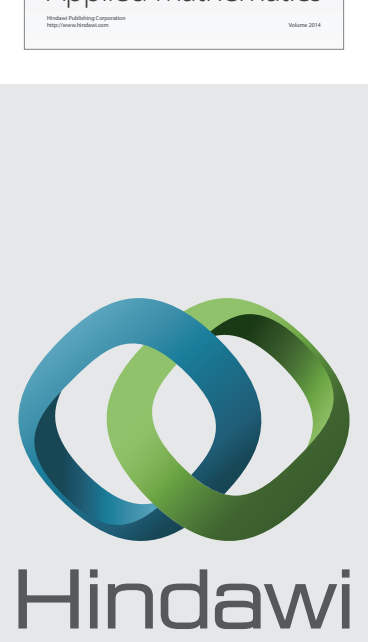

Submit your manuscripts at http://www.hindawi.com
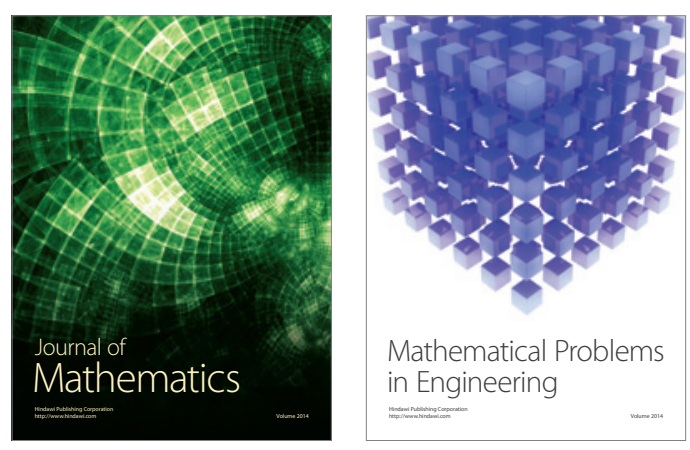

Mathematical Problems in Engineering
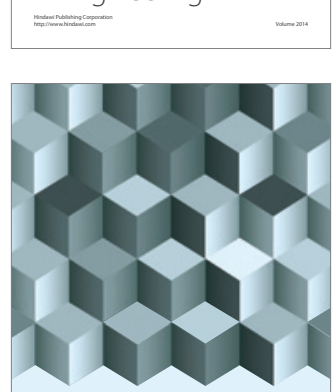

Journal of

Function Spaces
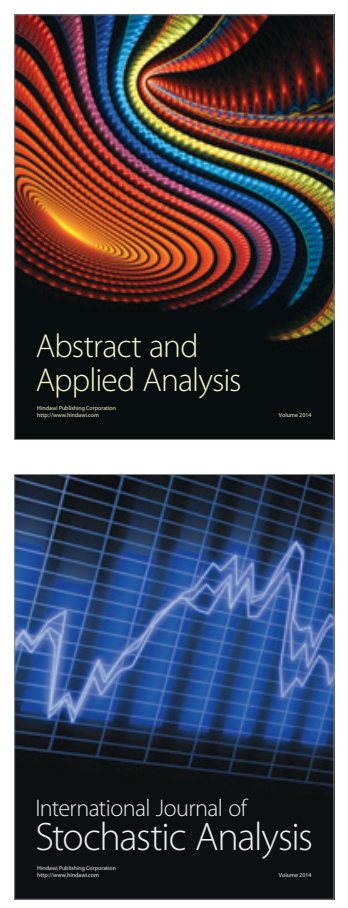

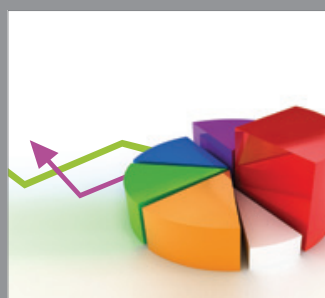

ournal of

Probability and Statistics

Promensencen
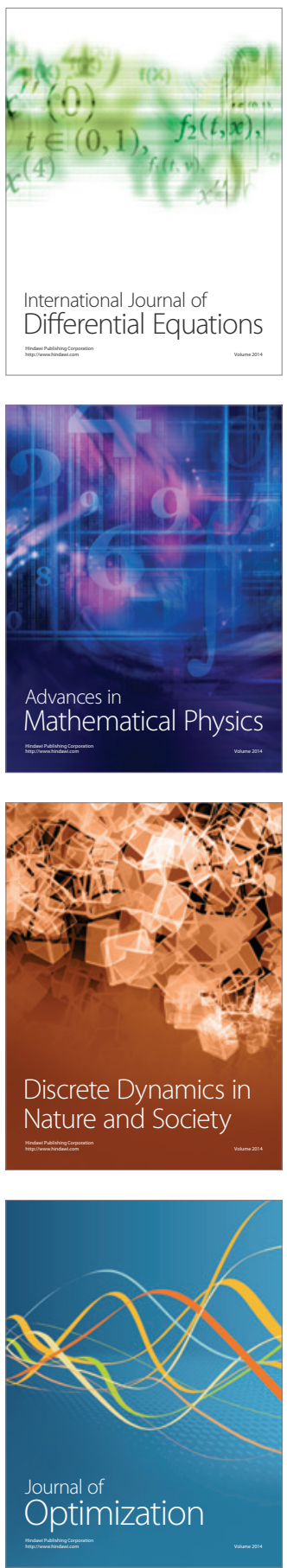